\title{
Investigation of Epigenetic Effects of Disulfiram on the Demethylation of RASSF1A Gene's Promoter in Breast Cancer Cell Line MCF-7
}

\author{
Leila Noori ${ }^{1,{ }^{*}}$, Mohsen Pourghasem ${ }^{2}$, Zahra Babazadeh ${ }^{3}$, Soraya \\ Khafri ${ }^{4}$, Davood Saboor ${ }^{5}$ \\ ${ }^{1}$ Babol University of Medical Sciences, Babol, Iran \\ ${ }^{*}$ Corresponding Author: Leila Noori, Babol University of Medical Sciences, Babol, \\ Iran.E-mail:eris_leili@yahoo.com
}

DOI: $10.21859 /$ mci-supp- 67

\section{Keywords:}

Epigenetic

RASSF1A Gene

DSF Drug

Breast Cancer

Methylation

\begin{abstract}
Introduction: Breast cancer is the most common type of cancer among women with very high mortality rates in the world. Genetic and epigenetic studies for early detection and treatment are important and vital in this way. In this regard, in order to assess the RASSF1A promotor methylation in breast cancer cell line, this research was conducted.

Materials and Methods: Remarkably, observing the unmethylated band in promoter of RASSF1A gene in breast treated cancerous cell line of MCF-7 with DSF by MSP method was one of the most important purpose of this project. The breast cancerous cell line of MCF-7 were treated for 1 and 3 days with DSF in different dozes ( 2.5 up to $37.5 \mu l)$ in triplicate repetition in order to find the IC50 doze.

Results: Correspondingly, the results of MTT method indicated that the cancerous cell line of MCF-7 which were treated with 5, 10, 12.5 and $15 \mu \mathrm{l}$ with DSF, had 40 to 60 percent alive cells. In treatment for 3 days, the dozes of 5, 10, 12.5 and $15 \mu l$ had methylated and unmethylated bands.Considerably, the treatment for 3 days, was partial methylated. For treatment of 1 day, the dozes 5, 10, 12.5 and $15 \mu$ were shown the methylated band and only the unmethylated band was observed in doze of $15 \mu \mathrm{l}$.It was concluded that the partial methylated was existed in treatment for one day in doze of $15 \mu \mathrm{l}$.

Conclusions: Consequently, It seems that the DSF needs more than 3 days treatment or high dozes for demethylation of the promotor of RASSF1A in breast's cancerous cell line MCF-7.
\end{abstract}

